\title{
Putting the "E" in SPIDER: Evolving Trends in the Evaluation of Environmental Enrichment Efficacy in Zoological Settings
}

\author{
Christina Alligood $^{1 *}$ and Katherine Leighty ${ }^{1}$ \\ ${ }^{1}$ Disney's Animal Kingdom \\ *Corresponding author (Email: Christina.Alligood@ disney.com)
}

Citation - Alligood, C., \& Leighty, K. (2015). Putting the "E" in SPIDER: Evolving trends in the evaluation of environmental enrichment efficacy in zoological settings. Animal Behavior and Cognition, 2(3), 200-217. doi: 10.12966/abc.08.01.2015

\begin{abstract}
In their seminal paper on environmental enrichment, Mellen and MacPhee (2001) proposed a set of broad goals for enrichment in zoological settings, as well as a framework for enrichment programs. Since that time, the philosophy and practice of environmental enrichment in zoos has continued to grow. Here we review evaluations of enrichment efficacy in the literature since 2001, looking for trends in species, target behaviors, enrichment strategies, and analytic techniques and discussing progress toward the SPIDER vision and outstanding needs in the field. We selected 94 peer-reviewed and 121 non-peer-reviewed articles for review, representing enrichment strategies across a wide range of species. The number of peer-reviewed articles published per year was relatively stable, such that the cumulative number of articles has continued to rise over the thirteen-year review period. We echo the call issued by a number of authors for continued and improved evaluation of enrichment efficacy, and add a recommendation for further exploration of single-subject experimental designs. We also call for focus on a broader array of species and on specific areas of application including reintroduction.
\end{abstract}

Keywords - Environmental enrichment, Zoos, Animal welfare

The study of environmental enrichment for animals in zoological settings has grown tremendously over the past several decades. Shepherdson (2003) gave a detailed definition of enrichment:

Environmental enrichment is a process for improving or enhancing zoo animal environments and care within the context of their inhabitants' behavioral biology and natural history. It is a dynamic process in which changes to structures and husbandry practices are made with the goal of increasing the behavioral choices available to animals and drawing out their species-appropriate behaviors and abilities, thus enhancing their welfare. As the term implies enrichment typically involves the identification and subsequent addition to the zoo environment of a specific stimulus or characteristic that the occupant(s) need but which was not previously present. (p.119)

In their seminal paper on environmental enrichment in 2001, Mellen and MacPhee proposed a set of broad goals for enrichment in zoological settings, as well as a framework for enrichment programs. Since that time, the philosophy and practice of environmental enrichment in zoos has continued to grow. Enrichment is now widely viewed as a critical component of animal care programs (Hutchins \& Smith, 2003) designed 
to maximize animal welfare (Barber, 2009) through a wide variety of strategies (Maple, 2007). Shepherdson (2003) traced the development of the concept of environmental enrichment into a field of its own:

However, environmental enrichment, in its current incarnation at least, is more than an arbitrary set of objects and activities deemed by the user to be 'effective' based on untested preconceptions. Enrichment is also a field of scientific study with its own set of guiding principles and underlying theoretical concepts. (p. 119)

The six-step framework for effective enrichment programs presented by Mellen and MacPhee (2001) includes Setting Goals, Planning, Implementing, Documenting, Evaluating, and Readjusting. The model has become affectionately known as "SPIDER" and the components are widely accepted as critical to the development and sustainment of state-of-the art programs. This is evident in the AZA accreditation standards (Association of Zoos and Aquariums 2015) language on enrichment programs:

1.6.1. The institution must have a formal written enrichment and training program that promotes species-appropriate behavioral opportunities, and a training program that facilitates husbandry and veterinary procedures where appropriate.

Explanation: An enrichment and training program should be based on current information in biology, and should include the following elements: goal-setting, planning and approval process, implementation, documentation/record-keeping, evaluation, and subsequent program refinement. The enrichment program should also apply to animals in quarantine, as appropriate and possible. Some birds, fish, reptiles, amphibians, and invertebrates may not require enrichment beyond the natural or social environment provided in their enclosure.

1.6.2. The institution must have a specific staff member(s) or committee assigned for enrichment program oversight, implementation, training, and interdepartmental coordination of enrichment efforts. (p. 10)

Foundational training in the implementation of enrichment programs has been accomplished by many zoos through the AZA professional development program's Managing Animal Enrichment and Training Programs (MAETP) course and other courses and workshops. Melfi and Hosey (2011) described a workshop on behavioral husbandry topics, including enrichment, designed to provide leadership training in these areas at smaller international zoos.

Specifically regarding the evaluation component of SPIDER, Mellen and MacPhee (2001) noted that systematic evaluations were at that time the exception rather than the rule and cautioned that subjective assessments are not sufficient for determining the effectiveness of enrichment strategies, pointing to the need to validate rapid assessment methods. In 2006, Barber noted that there were still "three-legged SPIDERS" (missing the "-DER") in existence and issued a reminder of the importance of implementing all components of the model in order to ensure effectiveness. Indeed, a number of authors have issued calls for continued and improved evaluation of enrichment efficacy (e.g., Kanaan \& Hötzel, 2011; Rosier \& Langkilde, 2011a, 2011b; Shepherdson, 2003). For example, after assessing the natural history of the species, Rosier and Langkilde (2011a) added items (i.e., raised basking platforms) to an eastern fence lizard's enclosure designed to meet the animal's behavioral needs. Subsequent observations revealed that the provision of these items did not have a significant effect on the animal's activity budget. The authors characterize this result not as an indication that environmental enrichment in general is unnecessary or ineffective, but as an indication of the need to carefully evaluate the effectiveness of enrichment strategies so that, if the intended results are not realized, alternative strategies can be devised. Here we review evaluations of enrichment efficacy in zoological settings in the years since Mellen and MacPhee's (2001) SPIDER framework was proposed, looking for trends in species, target behaviors, enrichment strategies, and analytic techniques and discussing progress toward the SPIDER vision and outstanding needs in the field. 
Bloomsmith (2009), in her report on an important animal welfare conference held at the Brookfield Zoo in Chicago in 2008, noted that animal-welfare research conducted in farm and laboratory settings is better established than research conducted in zoos, and advocated that zoo researchers seek input from and engage in dialog with researchers working in these other arenas. We agree with these points and believe that they extend to the subset of animal-welfare research focused on environmental enrichment. We will return to some extensions of these points later in the paper, but will focus our review on evaluations of environmental enrichment in zoos because we are specifically interested in the progress and development of work in this setting.

\section{Method}

To examine the study of enrichment evaluation in zoos since the inception of the SPIDER model (Mellen \& MacPhee, 2001), we conducted an issue-by-issue search from 2002 through 2014 of Zoo Biology, Applied Animal Behaviour Science, Animal Welfare, Journal of Applied Animal Welfare Science, Journal of Comparative Psychology, International Journal of Comparative Psychology, International Zoo Yearbook, and Journal of Zoo and Aquarium Research. We also completed an Academic OneFile database search for the same years, using the keywords "environmental enrichment" and "zoo". We selected articles that reported a direct evaluation, conducted at a zoological institution, of the effect of an enrichment strategy on one or more specific behavioral variables. There were relatively few articles on marine mammal behavior in the targeted journals. We excluded marine mammal species from our review so as not to misrepresent that important area of work by generalizing from this zoo-focused search.

Mellen and MacPhee (2001) suggested some broad goals for enrichment: enhancing animal welfare, successful reproduction, reducing stress, decreasing aberrant behavior and increasing speciestypical behavior, and successful reintroduction. We reviewed the stated goals of the enrichment strategies evaluated in the literature and compared them to these broad goals, looking for opportunities for enhanced focus in the coming years.

Though we applied the selection criteria above to articles in the peer-reviewed literature, we recognize that this literature may only represent a subset of the creative and innovative enrichment ideas implemented by zoo animal care staff. We wanted to get a sense of the scope and breadth of those ideas as well. To that end, we conducted an issue-by-issue search of Animal Keepers' Forum (AKF,) the official professional journal of the American Association of Zoo Keepers, for the years 2002-2014. The AKF publishes a monthly column, "Enrichment Options", describing environmental enrichment ideas and programs. The journal also regularly publishes articles on the philosophy of enrichment and evaluations of enrichment initiatives. Many AKF articles are authored by zoo keepers, a community noted by other authors as a rich source of information gleaned from many hours of animal observations in the course of daily care (e.g., Law \& Reid, 2010; Shepherdson, 2003). Other sources of similar information not reviewed here include The SHAPE of Enrichment newsletter, and international publications Ratel and International Zoo News.

\section{Results}

In our review of the scientific peer-reviewed literature, we selected 94 enrichment evaluation articles from 12 different journals. Four journals (Zoo Biology, Applied Animal Behaviour Science, Journal of Applied Animal Welfare Science, and Animal Welfare) accounted for the majority of articles; the remaining eight journals accounted for one or two articles each (see Table 1). The number of articles published per year was relatively stable (Range $=4-12$, Mean $=7, \mathrm{SD}=2$, Median $=7$, Mode $=7$ ), such that the cumulative number of articles has continued to rise over the thirteen-year review period (see Figure 1 ). The species groups represented in these articles included primates ( 20 species, 34 articles, $36 \%)$, felines (19 species, 20 articles, 21\%), bears ( 6 species, 12 articles, 13\%), canines ( 4 species, 8 articles, $8 \%$ ), reptiles and amphibians (9 species, 7 articles, $7 \%$ ), avians (7 species, 7 articles, $7 \%$ ), hoofstock ( 4 species, 3 articles, 
$3 \%$ ), large mammals ( 2 species, 2 articles, $2 \%$ ), and other mammals (7 species, 7 articles, $7 \%$ ), with some articles including more than one species or species group (see Table 2).

Table 1

Number of Selected Zoo Enrichment Evaluation Articles per Journal, 2002-2014

\begin{tabular}{lc}
\multicolumn{1}{c}{ Journal } & $\begin{array}{c}\text { Zoo Enrichment Evaluation } \\
\text { Articles, 2002-2014 }\end{array}$ \\
\hline Zoo Biology & 19 \\
Journal of Applied Animal Welfare Science & 16 \\
Applied Animal Behaviour Science & 16 \\
Animal Welfare & 2 \\
International Journal of Comparative Psychology & 2 \\
Journal of Zoo and Aquarium Research & 2 \\
Journal of Comparative Psychology & 1 \\
International Zoo Yearbook & 1 \\
American Journal of Primatology & 1 \\
Zoologia & 1 \\
Revista Cientifica & 1 \\
Animal Cognition & \\
TOTAL articles selected & 94 \\
\hline
\end{tabular}

Table 2

Focal Species in Selected Zoo Enrichment Evaluation and Device Description Articles, 2002-2014

\begin{tabular}{lcc}
\hline \multicolumn{1}{c}{ Focal Species } & $\begin{array}{c}\text { Peer-Reviewed Zoo Enrichment } \\
\text { Evaluation Articles }(n=94)\end{array}$ & $\begin{array}{c}\text { AKF Enrichment Evaluation and } \\
\text { Device Description Articles }(n=121)\end{array}$ \\
\hline Primates & $34(36 \%)$ & $31 \%$ \\
Felines & $20(21 \%)$ & $11 \%$ \\
Bears & $12(13 \%)$ & $11 \%$ \\
Canines & $8(8 \%)$ & $4 \%$ \\
Reptiles and amphibians & $7(7 \%)$ & $18 \%$ \\
Avian & $7(7 \%)$ & $11 \%$ \\
Hoofstock & $3(3 \%)$ & $7 \%$ \\
Large mammals & $2(2 \%)$ & $17 \%$ \\
Other mammals (armadillo, anteater, peccary, & $7(7 \%)$ & \\
wombat, two-toed sloth, river otters) & & \\
Note: Several articles included more than one species category; thus the number of articles sums to $>n$ and the percent of articles \\
including each species group sums to $>100$.
\end{tabular}




\section{Cumulative Zoo Enrichment Evaluation Articles 2002-2014}

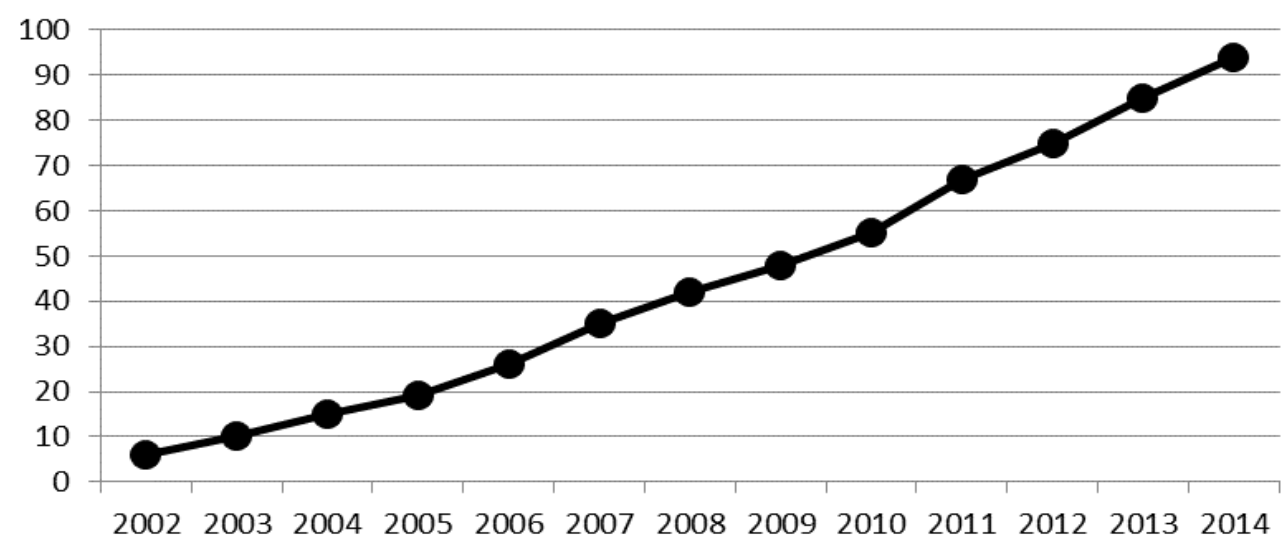

Figure 1. Cumulative number of selected peer-reviewed zoo enrichment evaluation articles, 2002 - 2014.

A variety of enrichment goals were set for these animals, including decreasing stereotypy (48 articles, 51\%), increasing activity (34 articles, 36\%), increasing species-typical behavior (including play; 26 articles, $28 \%$ ), increasing exploration or use of space (15 articles, 16\%), decreasing aggression (13 articles, 14\%), increasing behavioral choice or variety (10 articles, 11\%), increasing social interaction (6 articles, $6 \%$ ), and increasing reproductive success ( 2 articles, $2 \%$ ), with many articles including more than one enrichment goal (see Table 3). The enrichment strategies implemented in pursuit of these goals included foraging challenges (37 articles, 39\%), structural exhibit design modifications (15 articles, 16\%), manipulanda (14 articles, 15\%), olfactory stimuli (12 articles, 13\%), spatially-dispersed feeding (12 articles, $13 \%)$, temporally-dispersed feeding (11 articles, 12\%), naturalistic materials (9 articles, 10\%), auditory stimuli ( 8 articles, $8 \%$ ), other animals ( 8 articles, $8 \%$ ), choice of areas (5 articles, 5\%), audiovisual technology (5 articles, 5\%), positive-reinforcement training (4 articles, 4\%), novel foods (4 articles, $4 \%$ ), and visual blocking ( 3 articles, $3 \%$ ), with many articles including more than one enrichment strategy (see Table 4).

Table 3

Enrichment Goals in Selected Zoo Enrichment Evaluation Articles, 2002 - 2014

\begin{tabular}{lcc}
\hline \multicolumn{1}{c}{ Enrichment Goal } & $\begin{array}{c}\text { Peer-Reviewed Zoo } \\
\text { Enrichment Evaluation } \\
\text { Articles }(n=94)\end{array}$ & $\begin{array}{c}\text { AKF Enrichment } \\
\text { Evaluation Articles } \\
(n=121)\end{array}$ \\
\hline Decrease stereotypy & $48(51 \%)$ & $32 \%$ \\
Increase activity & $34(36 \%)$ & $8 \%$ \\
Increase species-typical behavior, including play & $26(28 \%)$ & $52 \%$ \\
Increase exploration or space use & $15(16 \%)$ & $20 \%$ \\
Decrease aggression & $13(14 \%)$ & $4 \%$ \\
Increase behavioral choice or variety & $10(11 \%)$ & $12 \%$ \\
Increase social interaction & $6(6 \%)$ & $8 \%$ \\
Increase reproductive success & $2(2 \%)$ & $0 \%$ \\
\hline
\end{tabular}

Note: Several articles included more than one enrichment goal; thus the number of articles sums to $>n$ and the percent of articles including each species group sums to $>100$. 
Table 4

Enrichment Strategies in Selected Zoo Enrichment Evaluation Articles, 2002 - 2014

\begin{tabular}{lcc}
\multicolumn{1}{c}{ Enrichment Strategy } & $\begin{array}{c}\text { Peer-Reviewed Zoo } \\
\text { Enrichment Evaluation } \\
\text { Articles, 2002-2014 }(n=94)\end{array}$ & $\begin{array}{c}\text { AKF Enrichment Evaluation } \\
\text { Articles }(n=121)\end{array}$ \\
\hline Foraging challenge & $37(39 \%)$ & $54 \%$ \\
Exhibit design modification & $15(16 \%)$ & $19 \%$ \\
Manipulanda & $14(15 \%)$ & $27 \%$ \\
Olfactory stimuli & $12(13 \%)$ & $23 \%$ \\
Spatially-dispersed feeding & $12(13 \%)$ & $8 \%$ \\
Temporally-dispersed feeding & $11(12 \%)$ & $0 \%$ \\
Naturalistic materials & $9(10 \%)$ & $19 \%$ \\
Auditory stimuli & $8(8 \%)$ & $0 \%$ \\
Other animals & $8(8 \%)$ & $4 \%$ \\
Choice of areas & $5(5 \%)$ & $0 \%$ \\
Audiovisual technology & $5(5 \%)$ & $0 \%$ \\
Positive-reinforcement training & $4(4 \%)$ & $8 \%$ \\
Novel foods & $4(4 \%)$ & $19 \%$ \\
Visual blocking & $3(3 \%)$ & $4 \%$ \\
Note: Several studies used multiple enrichment strategies; thus the number of articles sums to $>n$ and the percent of articles \\
including each species group sums to $>100$.
\end{tabular}

All studies included behavioral measures, and 10 studies (11\%) also included physiological measures such as fecal glucocorticoid metabolites. The number of subjects per study ranged from 1 to 48 . The upper limit of 48 was a clear outlier (the next-highest number of subjects was 29), so we removed it when calculating descriptive statistics on number of subjects $(M=7, S D=5$, median $=6$, mode $=4)$. Behavior was observed in sessions ranging from 1 to $1440 \mathrm{~min}$. Again, the upper limit was an outlier (the next-highest number of minutes was 720), so we removed it when calculating descriptive statistics on number of minutes per observation session $(M=107, S D=127$, median $=60$, mode $=60)$. Overall duration of each study ranged from 9 to 2190 days (about 6 years). The two longest studies were outliers; these studies observed behavior for four years and six years (the next-longest study was 23 months, or about 690 days). We removed these two when calculating descriptive statistics on study duration in days $(M=138$, $S D=124$, median $=90$, mode $=90)$. Most authors reported that enrichment strategies were effective in meeting at least some goals. Forty-one articles (44\%) reported effectiveness for all goals; forty-five (48\%) reported effectiveness for some but not others, and eight articles (8\%) reported that the implemented strategies were not effective). Most authors (88 articles, 94\%) used inferential statistics to evaluate their data, and many of those (39 articles, $44 \%$ ) issued a caveat regarding limited generality of results due to the small number of subjects studied.

In our review of non-peer-reviewed AKF enrichment articles, we selected 121 articles falling into three broad categories: descriptions of enrichment devices (46 articles); discussions of enrichment philosophy and/or program framework (50 articles); and evaluations of enrichment effectiveness (25 articles). In 2004, the Enrichment Options editors changed the format of the column to require that descriptions of enrichment devices include goal behaviors and documentation strategies (Cantrell \& Neptune, 2004).

Forty-five AKF articles described devices designed for environmental enrichment, and one article listed suppliers of commercially available devices. Many description articles gave step-by-step instructions for assembling enrichment devices. Several articles described devices for use across multiple taxa, and three articles described universal ideas for application with many different species. Devices were designed to 
promote natural behavior, decrease undesirable behavior, and/or provide stimulation and behavioral choice, with 34 articles (74\%) describing devices related to feeding.

Twenty-six articles evaluated the effectiveness of an enrichment strategy. Across description and evaluation articles, species groups represented included primates (22 articles, 31\%), avians (13 articles, $18 \%$ ), felines ( 8 articles, $11 \%)$, bears ( 8 articles, $11 \%)$, hoofstock ( 8 articles, $11 \%)$, large mammals $(5$ articles, $7 \%$ ), reptiles and amphibians (3 articles, 4\%), canines (1 article, 1\%), and other animals (small mammals, marsupials, bats; 12 articles, 17\%). Enrichment goals in evaluation articles included increasing species-typical behavior (13 articles, 52\%), decreasing stereotypy ( 8 articles, 32\%), increasing exploration or space use ( 5 articles, $20 \%$ ), increasing behavioral choice or variety ( 3 articles, $12 \%$ ), increasing social interaction ( 2 articles, $8 \%$ ), increasing activity ( 2 articles, $8 \%$ ), and decreasing aggression ( 1 article, $4 \%$ ). Strategies implemented included foraging challenges (14 articles, 54\%), manipulanda (7 articles, 27\%), olfactory stimuli (6 articles, $23 \%$ ), novel foods (5 articles, 19\%), structural exhibit modifications (5 articles, $19 \%$ ), natural materials (5 articles, 19\%), positive-reinforcement training (2 articles, 8\%), spatiallydispersed feeding ( 2 articles, $8 \%$ ), visual blocking ( 1 article, $4 \%$ ), and other animals ( 1 article, $4 \%$ ).

Fifty articles discussed the philosophy of environmental enrichment and/or frameworks for specific enrichment programs. Twenty-eight of these discussed enrichment programs for particular species or species groups, including primates, bears, large mammals, felines, avians, reptiles and amphibians.

\section{Discussion}

\section{Focal Species}

Both the scientific literature and the AKF enrichment articles included a wide variety of focal species, with some taxa more heavily represented than others (most notably primates and felines). The relatively high number of studies focusing on these species may be related to high rates of stereotypy (Mason, Clubb, Latham, \&Vickery, 2007). One notable difference between the two sets of articles was a lower percentage of AKF articles focusing on felines, and a greater percentage focusing on avians and a variety of mammals including river otters, bats, raccoons, kangaroos, and others, reflecting keeper ingenuity in creating enrichment strategies for a wide variety of species.

Some notable species-specific reviews of the enrichment literature have appeared in recent years (e.g., bears, Law \& Reid, 2010; tigers, Szokalski, Litchfield, \& Foster, 2012). As the field continues to target a variety of behavioral needs through enrichment strategies, we hope to see more prolific scientific work with a broad array of species. For example, enrichment strategies for reptiles and amphibians have been less often studied in laboratories as well as in zoos (Burghardt, 2013). This relative scarcity of studies represents an area of opportunity for investigating the needs of these animals and successful enrichment strategies for meeting those needs. As another example, Cloutier and Packard (2014), citing a dearth of studies on environmental enrichment for African painted dogs, conducted a survey of institutions participating in the African painted dog Species Survival Plan regarding the types, variety, and implementation of enrichment strategies. Respondents reported a wide variety of enrichment types in use, and the authors noted that individual variation in preferences could be a challenge when interpreting the few available enrichment evaluation studies for application with this species. Izzo, Bashaw, and Campbell (2011) also pointed to the need for more focus on individual differences. Tetley and O'Hara (2012) reviewed the literature on personality assessment with zoo-housed animals and recommended these assessments as a means of measuring individual differences particularly in relation to social and reproductive behavior.

\section{Enrichment Goals, Strategies, and Measures}

Mellen and MacPhee (2001) discussed the importance of matching enrichment goals and resulting strategies to animals' natural history, a concept also supported by other authors (e.g., Koene, 2013; Newberry, 1995; Shepherdson, 2003). It has become common practice to consider natural history not only 
when devising ad hoc environmental enrichment plans, but also when designing the structural elements of exhibits themselves. Both sets of articles in our review placed a heavy emphasis on natural history when discussing behavioral goals and the resulting enrichment strategies. The behavioral goals described in these studies led to the implementation of strategies designed to facilitate each behavior. Mellen and MacPhee (2001) provided five broad enrichment goals to guide development of more specific individualized goals. While there may be a need to critically evaluate these goals through the lens of animal welfare as the study of that area continues to progress, the original five goals form an outline for discussing the studies reviewed here in the context of the broader literature.

Goal 1: Enhancing animal welfare. Dawkins (2004) suggested a "two questions" approach to welfare that asks, "Are the animals healthy?" and "Do the animals have what they want?", with the second question being addressed through measurement of animals' preferences through their behavior. Balcombe (2009) argues that we have a moral obligation to learn more about the conditions under which nonhuman animals experience pleasure. For example, he describes play, tasting food, engaging in sexual/social interaction, and seeking comfort/homeostasis as behaviors that seem likely to be related to the experience of pleasure. The enrichment goals addressed in the literature reviewed here are certainly consistent with this reasoning, For example, the provision of behavioral choices and opportunities to engage in speciestypical behavior is designed to enhance animal welfare in part by facilitating pleasure-seeking behavior.

It has been suggested that cognitive challenges may provide welfare-enhancing enrichment (Meehan \& Mench, 2007) for great apes (Clark, 2011) and marine mammals (Clark, 2013; Harley, Fellner, \& Stamper, 2010) in particular. This idea remains largely untested. Placing cognitive challenges in the context of the SPIDER model may help with this process. Consistent with SPIDER, Meehan and Mench (2007) suggest selecting specific challenges based on animals' natural history and determining measures that can be used to evaluate the effects of the challenges. Morimura (2003) provided an example of a feeding enrichment designed to allow the option of tool use in chimpanzees in managed care, a category of behavior seen in the wild. The animals employed all available choices to access orange juice, including tool use as well as direct use of mouth and hands.

The human-animal relationship (HAR) has long been a topic of study in farm settings, and has recently received some consideration in the zoo setting as well, with some authors suggesting that interaction with humans, and positive reinforcement training (PRT) in particular, may serve as environmental enrichment for zoo-housed animals (e.g., Claxton, 2011; Westlund, 2014a, 2014b). Several authors have debated the merits of PRT as enrichment, generally concluding that both training and other enrichment strategies are required to achieve optimal welfare (Baker, Bloomsmith, Neu, Griffis, \& Maloney, 2010; Melfi, 2013, 2014). Claxton (2011) cited instances where the results of PRT were consistent with enrichment goals (e.g., reduction of aggressive behavior during feeding through PRT-facilitated cooperative feeding) as examples of PRT's potential for enrichment. For example, Pomerantz and Terkel (2009) studied the behavior of a group of 12 chimpanzees before and after the implementation of a PRT program, and found a decrease in abnormal behaviors and an increase in affiliate behaviors after the program began. Interestingly, these behavioral changes were not confined to training sessions but were observed throughout the day.

Measuring the effects of environmental enrichment on welfare requires the ability to measure aspects of welfare itself. Bloomsmith (2009) noted the potential of tools for the rapid assessment of animal welfare to enhance accreditation processes. Föllmi et al. (2007) presented a scoring system designed to evaluate quality of life in geriatric zoo mammals and assist with decision-making. Several welfare measurement tools based on work in farms and other settings, including specific measures of the HAR, may prove useful in zoo settings as well (Claxton, 2011; Scott, Fitzpatrick, Nolan, Reid, \& Wiseman, 2003; Scott, Nolan, Reid, \& Wiseman-Orr, 2007).

Some authors have proposed the use of cognitive bias tasks to monitor animals' well-being (e.g., Mendl, Burman, Parker, \& Paul, 2009; Mendl \& Paul, 2004;). In these tasks, researchers first train animals to make distinct responses to two different stimuli; one stimulus predicts a positive event, and the other stimulus predicts a less-positive event. They then measure responses to ambiguous, or neutral, stimuli following environmental events, such as the provision or removal of enrichment, as a means of assessing 
animals' affect, or "judgment" of those events. For example, Bateson and Matheson (2007) found that European starlings that had previously been housed in an enriched cage environment but were then moved to a standard cage were more likely to classify ambiguous stimuli as being associated with a negative outcome than were there counterparts who experienced other housing arrangements. Keen et al. (2014) investigated the use of a similar task with grizzly bears housed at a conservation center. The bears were trained to respond differentially to light and dark cards associated with quantitatively different food rewards. When presented with an ambiguous (intermediate shade) card, the bears did not show an "optimistic bias" after engaging with preferred enrichment items, but did show this bias after engaging in bouts of stereotypic pacing, which for this group of bears was known to be associated with upcoming feeding times. This was the first animal study to successfully use a cognitive bias task based on different reward quantities, and the first to use this type of assessment with a wild carnivore.

Goal 2: Successful reproduction. Carlstead and Shepherdson (1994) described three mechanisms through which enriched environments might facilitate successful reproduction: support for developmental adaptability via increased environmental complexity; modulation of stress and arousal; and modification of social interactions. In their discussion of enhancement of reproduction through husbandry training, Desmond and Laule (1994) suggested that outcomes of effective environmental enrichment such as increased species-typical behavior and decreased stress can support successful reproduction. From this perspective, many of the enrichment strategies reviewed here, while not specifically focused on successful reproduction, could have supportive effects. The panda captive breeding program discussed by Swaisgood, Zhou, Zhang, Lindberg, and Zhang (2003) is a classic example of the use of enrichment strategies to support successful reproduction indirectly.

Goal 3: Reducing stress. Fecal glucocorticoid metabolites (FGM) have been used as a measure of stress in several species (e.g., polar bears, Hawaiian honeycreepers, and Clouded leopards, Shepherdson, Carlstead, \& Wielebnowski, 2004; Canada lynx, Fanson \& Wielebnowski, 2013; African wild dogs, Rafacz $\&$ Santymire, 2014). We noted in our review whether FGM or another physiological measure was used in addition to behavioral measures; this was the case in $11 \%$ of the scientific evaluations. Typically these studies have not identified changes in hormone levels as an enrichment goal per se, but have correlated these changes with environmental events and used those correlations to recommend environmental elements that may result in fewer acutely stressful events. This important area of work includes many more studies than those reviewed here (e.g., Chosy, Wilson, \& Santymire, 2014; Jacobs et al., 2014; Kumar, Reddy, Kokkiligadda, Shivaji, \& Umapathy, 2014; Narayan, Webster, Nicolson, Mucci, \& Hero, 2013); we did not include them because we focused on direct evaluations of enrichment effectiveness.

There has also been considerable debate regarding the effect of zoo visitors on animal behavior and welfare, with some studies reporting neutral or positive effects and others reporting behavior indicative of increased stress (Claxton, 2011; Davey, 2007; Farrand, Hosey, \& Buchanan-Smith, 2014; Fernandez, Tamborski, Pickens, Timberlake, 2009; Hosey, 2000, 2005; Kuhar, 2008; Ross, Londsdorf, Stoinski, 2007; Smith \& Kuhar, 2010; Stoinski, Jaicks, Drayton, 2012; Wells, 2005). Davey (2007) suggested that more study is needed before strong conclusions may be drawn, and called on zoo staff to monitor stress indicators in animals as a means of assessing visitor effects at specific exhibits. Similarly, Kuhar (2008) and Stoinski et al. (2012) emphasized the importance of monitoring visitor effects on individual animals after finding inconsistent affects among several groups of gorillas. Fernandez et al. (2009) provided some specific and testable recommendations (involving zoo staffing, exhibit design, and enrichment strategies) for balancing the need to generate visitor interest with the potential adverse impacts of large crowds on animal welfare.

Goal 4: Decreasing aberrant behavior and increasing species-typical behavior. As the practice of setting enrichment goals specific to species and individuals has come into wider use, so has the practice of evaluating the success of enrichment strategies at meeting those specific goals. Siegford (2013) describes a number of behavior-based measures that may prove useful, including preference tests. In her view, measures of space and resource use in zoo exhibits are a form of preference testing. Fraser (2009) suggested thinking about maximizing welfare through naturalistic living conditions as a two-by-two table, where behavior might be either natural or not for a species and might be either preferred or not by an individual. Welfare might be maximized by accommodating behavior that is both natural and preferred. 
While interaction with or manipulation of an enrichment item is not in and of itself an indicator of enrichment effectiveness (Shyne, 2006), it may be a prerequisite for the success of many strategies. To investigate the circumstances under which this prerequisite occurs, some researchers have measured time spent interacting with an enrichment device. For example, Kuczaj et al. (2002) found that habituation effects could be minimized, and thus interaction maximized, when enrichment items were made available on a schedule in which both the time of day and the length of time (e.g., 1-15 min) were varied. Mehrkam and Dorey (2014) evaluated preference assessments as a predictor of enrichment efficacy in Galapagos tortoises and suggested that preference and effectiveness are sometimes, but not always, related. Ross, Schapiro, Hau, and Lukas (2009) used a preference measure called an electivity index to study the use of specific enclosure elements by great apes before and after a move to a new enclosure with naturalistic design elements. The electivity index measures the animals' use of different features of their environment in relation to that feature's relative availability. For example, if an animal spends a great deal of time resting under a tree and there is only one tree in the environment, the tree would be considered a preferred feature, and perhaps even over-used in comparison with other features. Ross et al. (2009) found that the electivity index was able to measure important changes in gorillas' and chimpanzee's use of available space following the move to their new enclosures.

Another instance in which interaction with an item is a useful measure is when the enrichment goal is to increase behavioral choice/variety. For instance, Maloney, Leighty, Kuhar, and Bettinger (2011) measured the rate at which gorillas watched videos depicting different gorilla and human activities, and also measured the gorillas' other behavioral responses to the videos, finding that the gorillas did choose to spend time watching the videos, and that they preferred to watch videos of gorillas engaging in agonistic behaviors. Schapiro and Lambeth (2007) recommended the adaptation of consumer-demand preference assessments to measure the relative value of resources to animals. They noted that to generate a complete picture, this kind of preference testing needs to be conducted across different times of day, in different seasons, with varying visitor numbers, etc. For example, Posta, Huber, and Moore (2013) conducted detailed observations of the behavior of two elephants across different times of day and across seasonal changes over a two-year period. Their results showed pronounced differences in preferred resources and activities across different times of day and seasons. For example, both elephants fed less and walked more at night than during the day, and spent more time walking during the day in the winter than in the summer. These differences underscore the need to consider temporal variations when measuring animal preferences.

Some authors have explored the extent to which zoos are offering particular categories of enrichment designed to provide opportunities for species-typical behavior. For example, Robins and Waitt (2011) reviewed the literature on the provision of access to water features as an enrichment strategy for macaques, and Huber and Lewis (2011) surveyed 46 zoos housing gummivorous primates (e.g., marmosets, tamarins, lorises, baboons), asking whether gum is provided to these animals. As zoological institutions continue to work toward providing environments that can best stimulate species-typical behavior in a broad range of species, this sort of multi-institution data gathering and subsequent prescriptive recommendations will be useful for other species as well.

Almost half of the studies in our review implemented strategies designed to reduce stereotypy. Mason and Latham (2004) and Mason et al. (2007) made a case for the serious consideration of stereotypy as a potential indicator of less-than-optimal welfare. Shepherdson, Lewis, Carlstead, Bauman, and Perrin (2013) studied stereotypic pacing in a large sample of zoo-housed polar bears through direct observation and found that 47 of 55 bears engaged in some pacing. Mason et al. (2007) estimated, through records review, a strikingly high prevalence of stereotypic behavior in several zoo animal populations. Shepherdson et al. (2013) identified environmental enrichment and training as possible mitigating factors for stereotypic pacing in polar bears. Shyne (2006) reviewed the literature on the effectiveness of enrichment strategies designed to reduce stereotypy and found that the majority of studies reported that stereotypy was reduced when enrichment strategies were implemented. Mason et al. (2007) noted, however, that no published studies evaluating enrichment designed to reduce stereotypy have reported $100 \%$ reductions in all subjects, suggesting that the selected enrichment strategies are not always successful and perhaps that the target behaviors have sometimes become resistant to change by the time the enrichment strategies are 
implemented. Mason and Latham (2004) and Mason et al. (2007) suggested that it is critical to investigate the underlying factors contributing to this behavior in individual animals (and the topography of the behavior itself may not always be a reliable indicator of its function). Based on this information, decisions can be made about the best strategies to maximize welfare in each situation. As noted by Mason et al. (2007), this approach is consistent with the applied behavior-analytic human treatment literature (e.g., Cunningham \& Schreibman 2008; Shabani, Wilder, \& Flood, 2001).

Bassett and Buchanan-Smith (2007) reviewed the literature on predictability of environmental events in relation to animal welfare and concluded that unpredictable feeding can lead to increased exploration and other species-typical behavior. However, unreliable signals (e.g., sounds, such as a door opening, that sometimes but do not always precede feeding) can lead to aggression and other signs of frustration. Based on these findings, the authors suggested that unpredictable feeding schedules should be used when possible, and that reliable signals for feeding (e.g., a buzzer) should be introduced.

Goal 5: Successful reintroduction. Only one of the studies reviewed here was specifically intended to enhance preparations for reintroduction (Coelho, Schetini de Azevedo, \& Young, 2012). Many of the goals of environmental enrichment are consistent with the skills needed for survival in the wild. For example, enrichment strategies that promote species-typical behavior such as foraging, shelter-building or seeking, and successful reproduction in pre-release settings may, in turn, increase the likelihood of those behaviors occurring in post-release settings and ultimately enhance the rate of survival. Enrichment has thus become an important part of reintroduction programs. Reading, Miller, and Shepherdson (2013) noted that historically, most reintroduction attempts have failed and there is much work to be done to improve the success of such attempts, particularly in light of the global biodiversity crisis. It is therefore critical that we develop good methods for evaluating the effectiveness of enrichment strategies designed to support natural behaviors that allow animals to successfully transition to wild habitats.

\section{A Note on Visitor Perception}

One goal that falls outside the animal-welfare-focused enrichment framework but is nevertheless important to zoological institutions is visitor perceptions of animals, animal exhibits, and of the institutions themselves. Some authors (e.g., Hare et al., 2003; Kreger, Hutchins, \& Fascione, 1998) have expressed concern that un-naturalistic enrichment might detract from visitor experiences at naturalistic exhibits, but there is evidence that zoo visitors have some awareness of the value of environmental enrichment (Reade $\&$ Waran, 1996). A number of studies investigating visitor interest and perceptions of animal welfare and educational messages at exhibits with varying degrees of naturalistic design and enrichment have reported that visitor perceptions are less related to the type of enrichment item and more related to the degree of naturalism in the design of the exhibit itself (e.g., Davey, 2006; Davey, Henzi, \& Higgins, 2005;; Kutska, 2009; McPhee, Foster, Sevenich, \& Saunders, 1998). In addition, Miller (2012) found that visitor perception of animal care and interest in supporting zoos both declined after the visitors viewed a tiger pacing. These results further support the high value of environments designed to meet behavioral needs, even when those environments include some non-naturalistic elements.

\section{Methods of Analysis}

Zoo keepers have a vested interest in developing and providing effective enrichment strategies for the animals in their care, and have long been vocal advocates for enrichment as a critical part of husbandry programs (Shepherdson, 2003). Several authors have pointed to the value of keeper-conducted behavioral observations but noted the challenges of collecting behavioral data within a daily keeper schedule (Canino \& Powell, 2010; Hoy, Murray, \& Tribe, 2010a; Margulis \& Westhus, 2008; Quirke \& O'Riordan, 2013; Shyne, 2006). The 60-min median observation session duration in the studies reviewed here is quite a long period to fit within a keeper's routine. Hoy et al. (2010a) conducted a survey regarding enrichment for captive mammals; their respondents were 238 professionals from 60 zoos in 13 countries. Respondents reported that while they considered the provision of different kinds of enrichment (e.g., feeding, structural, 
social, auditory) to be important, only those modalities that were least time-consuming for the staff were frequently provided. Most respondents reported using a formal enrichment approval process at least some of the time. About half of respondents indicated that regular documentation or formal recording of enrichment data never took place, and a lack of available staff time for evaluation was the most frequently reported factor limiting the effectiveness of enrichment. Most respondents reported that more evaluation would be carried out if it were manageable.

Canino and Powell (2010) conducted a brief study of polar bear behavior to determine the utility of evaluating enrichment strategies using brief observations conducted by keepers during their work routines. The authors were able to observe changes in the bear's activities over time and as enrichment strategies shifted. They did not directly compare the results obtained with this data collection method to other, more time intensive methods. Margulis and Westhus (2008) and Quirke and O'Riordan (2013) each compared sampling periods of differing lengths and concluded that while brief point-scan observations did not detect accurate estimates of rare behaviors, they did provide estimates comparable with more intensive methods for more common behaviors (those occurring during over $15 \%$ of the intervals in an activity budget) and could therefore be useful in certain contexts. Quirke and O'Riordan (2013) also noted that in many cases, several brief (e.g., $15 \mathrm{~min}$ ) observations adding up to $60-120 \mathrm{~min}$ per day might be effective for evaluation. Shyne (2006) noted the importance of continual monitoring of enrichment effectiveness by zoo staff because many published studies only measure effects over a short time. This was true of the studies reviewed here, most of which measured effects over about three months.

In most of the evaluations in our review, the number of subjects was quite small. Indeed, many authors remarked on this when discussing their results. In their GAP analysis of the environmental enrichment literature, Schetini de Azevedo et al. (2007) reported finding 62 enrichment studies conducted in zoos between 1985 and 2004. The average number of subjects in these studies was nine, roughly consistent with the mean of seven subjects in the articles reviewed here. The population of animals of a particular species at any one zoo is often small enough to pose challenges for researchers attempting to use inferential statistics to support conclusions about the general population. Several researchers have recommended larger multi-institutional studies (e.g., Koene, 2013; Shepherdson et al., 2013; Swaisgood, 2007) with sample sizes large enough to meet the assumptions required for these tests (e.g., Shepherdson, 2003; Swaisgood \& Shepherdson, 2005). We certainly agree that researchers should take care to ensure that the tests they use are appropriate to their questions, their methods, and their data. It is also worthwhile to consider the importance of multiple categories of questions under the umbrella of environmental enrichment. One such category involves conclusions about a general population. For example, Swaisgood and Shepherdson (2005) suggest two goals for enrichment research in zoos: prediction of the circumstances under which stereotypies are most likely to develop, and prediction of the types of enrichment strategies required to mitigate stereotypies across various taxa and circumstances. Multi-institutional studies are an excellent means of addressing these questions, and there have been some notable examples in recent years (e.g., Posta, Leighty, Alligood, \& Carlstead, 2013; Shepherdson et al., 2004, 2013). It is equally important to build a knowledge base that will allow us to determine, for example, the cause(s) of stereotypy for a particular polar bear and use that information to design environments to support species-typical behavior and measure effectiveness for that individual. Both levels of analysis are necessary if we are to be successful in using environmental enrichment to promote optimal animal welfare. Because welfare is at the individual level, the processes used every day by keepers and other animal care staff require careful attention and refinement.

Fortunately, null-hypothesis significance testing is not the only means of assessing the effects of independent variables on behavior. The field of applied behavior analysis (Baer, Wolf, \& Risley, 1968; 1987) has a rich history of employing single-subject experimental designs to study both human and nonhuman animal behavior in laboratory and applied settings. A central goal of applied behavior analysis is to investigate methods of assessing the impact of the environment on an individual's behavior and then adjusting the environment to change behavior in ways that are beneficial to the individual. While different environmental factors may affect each individual's behavior, in this type of work each individual analysis serves as a replication of the analytic method itself (Johnston, 1979; Sidman, 1960). There have been calls 
in recent years for exploration of the application of this area to the behavior of animals in zoos (e.g., Bloomsmith, Marr \& Maple, 2007; Maple, 2007; Tarou \& Bashaw, 2007), and some examples of animal applications in other settings are available (Edwards \& Poling, 2011). Given the need for data-based decision making in the day-to-day implementation of environmental enrichment with individual animals, this area of work seems ripe for application of behavior-analytic methodology.

\section{The Next Frontier}

Enrichment technology. Hoy, Murray, and Tribe (2010b) suggested that, in the future, use of automated enrichment systems with Radio-frequency identification (RFID) technology might be one way to address the scarcity of staff time for providing and evaluating enrichment. They surveyed 233 professionals from 60 zoos in 13 countries and found that most respondents were interested in the development of this type of enrichment technology, for a variety of reasons (e.g., providing unpredictability and variety; allowing access to enrichment even when staff is unavailable; customizing enrichment for individuals based on microchip identification). Clay, Perdue, Gaalema, Dolins, and Bloomsmith (2011) found that the overall use of technology in enrichment programs is increasing (also see Pepperberg, 2004). These authors surveyed a small sample of zoological institutions housing primates and reported a variety of different technologies used in enrichment programs, the most common of which were computers, television, radio, and sprinklers. Clay et al. (2011) suggested that technology could be used to increase behavioral diversity, increase control and choice, and create a more complex environment. The most common reported problems with technological enrichment devices stemmed from the fact that, in most instances, the devices were designed for human use. Few respondents reported systematically evaluating the effectiveness of these enrichment devices; this will be needed as technology use increases.

A scientific foundation. Maple $(2007,2013)$ suggested that zoological institutions with a scientific foundation are best placed to provide optimal welfare for animals in their care. Lawson, Ogden, and Snyder (2008) examined prevailing attitudes toward science in zoological institutions and found that, despite an acknowledgment of the importance of science, negative perceptions of science and scientists persisted. Lawson et al. (2008) suggest that this can be mitigated, in part, by balancing scientific pursuits with the art of animal management as well as other institutional concerns. We suggest that the development of day-today enrichment evaluation methods to be employed by animal care staff might be an example of this balance. Law and Reid (2010) also spoke to this concern, emphasizing the symbiotic relationship between scientists and animal care staff.

When zoo animal care staff and scientists are directly involved in assessing enrichment effectiveness, we are able to directly advocate for management practices consistent with our findings (Bloomsmith, 2009). As the practice of environmental enrichment continues to evolve, some authors have predicted that the current terminology will fall out of favor. Barber (2006) predicted that "environmental" enrichment will evolve into a piece of a broader welfare framework. Hutchins and Smith (2003) suggested:

As recommended by Shepherdson et al. [1998], these enrichment activities will present captive animals with cognitive challenges, allow opportunities for appropriate social interaction and exploration, give the animals some control over their environment, and meet species-specific behavioural needs through the provision of shelter, hiding, foraging, and exercise. As such considerations are fully incorporated into exhibit design, the term 'environmental enrichment' will become passé. (p. 135)

We celebrate the tremendous growth of the field of environmental enrichment in zoological settings, and applaud the progress made in evaluating the effectiveness of many enrichment strategies. In the coming years, we look forward to continued innovation and development of fully-integrated environmental enrichment programs, along with more refined methods to assist with data-based day-to-day decision making in those programs. 


\section{Acknowledgements}

We thank Emily Boyd, Adam Ramsey, and Chelsea Weibel for assistance with the review of many articles, and Michelle Skurski and Angela Miller for insightful discussions of some of the topics presented here, and Jill Mellen for helpful comments on an earlier version of the manuscript.

\section{References}

Association of Zoos and Aquariums (2015). The accreditation standards and related policies: 2015 Edition. Online at https://www.aza.org/accreditation.

Baer, D. M., Wolf, M. M., \& Risley, T. R. (1968). Some current dimensions of applied behavior analysis. Journal of Applied Behavior Analysis, 1, 91-97.

Baer, D. M., Wolf, M. M., \& Risley, T. R. (1987). Some still-current dimensions of applied behavior analysis. Journal of Applied Behavior Analysis, 20, 313-327.

Baker, K. C., Bloomsmith, M. A., Neu, K., Griffis, C., \& Maloney, M. (2010). Positive reinforcement training as enrichment for singly housed rhesus macaques (Macaca mulatta). Animal Welfare, 19, 307-313.

Balcombe, J. (2009). Animal pleasure and its moral significance. Applied Animal Behaviour Science, 118, $208-216$.

Barber, J. (2006). Enrichment is dead! Animal Keepers' Forum, 33, 162-164.

Barber, J. C. E. (2009). Programmatic approaches to assessing and improving animal welfare in zoos and aquariums. Zoo Biology, 28, 519-530.

Bassett, L., \& Buchanan-Smith, H. M. (2007). Effects of predictability on the welfare of captive animals. Applied Animal Behaviour Science, 102, 223-245.

Bateson, M., \& Matheson, S. M. (2007). Performance on a categorisation task suggests that removal of environmental enrichment induces 'pessimism' in captive European starlings (Sturnus vulgaris). Animal Welfare, 16, 3336.

Bloomsmith, M. A. (2009). Measuring zoo animal welfare. Journal of Applied Animal Welfare Science, 12, $273-275$.

Bloomsmith, M. A., Marr, M. J., \& Maple, T. L. (2007). Addressing nonhuman primate behavioral problems through the application of operant conditioning: Is the human treatment approach a useful model? Applied Animal Behaviour Science, 102, 205-222.

Burghardt, G. M. (2013). Environmental enrichment and cognitive complexity in reptiles and amphibians: Concepts, review, and implications for captive populations. Applied Animal Behaviour Science, 147, 286-298.

Canino, W., \& Powell, D. (2010). Formal behavioral evaluation of enrichment programs on a zookeeper's schedule: A case study with a polar bear (Ursus Maritimus) at the Bronx Zoo. Zoo Biology, 29, 503-508.

Cantrell, R., \& Neptune, D. (2004). The new and improved enrichment options column. Animal Keepers' Forum, 31, 25-26.

Carlstead, K., \& Shepherdson, D. (1994). Effects of environmental enrichment on reproduction. Zoo Biology, 13, 447458.

Chosy, J., Wilson, M., \& Santymire, R. (2014). Behavioral and physiological responses in felids to exhibit construction. Zoo Biology, 33, 267-274.

Clark, F. E. (2011). Great ape cognition and captive care: Can cognitive challenges enhance well-being? Applied Animal Behaviour Science, 135, 1-12.

Clark, F. E. (2013). Marine mammal cognition and captive care: A proposal for cognitive enrichment in zoos and aquariums. Journal of Zoo and Aquarium Research, 1, 1-6.

Claxton, A. M. (2011). The potential of the human-animal relationship as an environmental enrichment for the welfare of zoo-housed animals. Applied Animal Behaviour Science, 133, 1-10.

Clay, A. W., Perdue, B. M., Gaalema, D. E., Dolins, F. L., \& Bloomsmith, M. A. (2011). The use of technology to enhance zoological parks. Zoo Biology, 30, 487-497.

Cloutier, T. L., \& Packard, J. M. (2014). Enrichment options for African painted dogs. Zoo Biology, 33, 475-480.

Coelho, C. M., Schetini de Azevedo, C., \& Young, R. J. (2012). Behavioral responses of maned wolves (Chrysocyon brachyurus, Canidae) to different categories of environmental enrichment stimuli and their implications for successful reintroduction. Zoo Biology, 31, 453-469.

Cunningham, A. B., \& Schreibman, L. (2008). Stereotypy in autism: The importance of function. Research in Autism Spectrum Disorders, 2, 469-479.

Davey, G. (2006). Relationships between exhibit naturalism, animal visibility and visitor interest in a Chinese zoo. Applied Animal Behaviour Science, 96, 93-102. 
Davey, G. (2007). Visitors' effects on the welfare of animals in the zoo: A review. Journal of Applied Animal Welfare Science, 10, 169-183.

Davey, G., Henzi, P., \& Higgins, L. (2005). The influence of environmental enrichment on Chinese visitor behavior. Journal of Applied Animal Welfare Science, 8, 131-140.

Dawkins, M. S. (2004). Using behavior to assess animal welfare. Animal Welfare, 13, S3-S7.

Desmond, T., \& Laule, G. (1994). Use of positive reinforcement training in the management of species for reproduction. Zoo Biology, 13, 471-477.

Edwards, T. L., \& Poling, A. (2011). Animal research in the Journal of Applied Behavior Analysis. Journal of Applied Behavior Analysis, 44, 409-412.

Fanson, K. V., \& Wielebnowski, N. C. (2013). Effect of housing and husbandry practices on adrenocortical activity in captive Canda lynx (Lynx canadensis). Animal Welfare, 22, 159-165.

Farrand, A., Hosey, G., \& Buchanan-Smith, H. (2014). The visitor effect in petting zoo-housed animals: Aversive or enriching? Applied Animal Behaviour Science, 151, 117-127.

Fernandez, E. J., Tamborski, M. A., Pickens, S. R., \& Timberlake, W. (2009). Animal-visitor interactions in the modern zoo: Conflicts and interventions. Applied Animal Behaviour Science, 120, 1-8.

Föllmi, J., Steiger, A., Walzer, C., Robert, N., Geissbühler, U., Doherr, M. G., \& Wenker, C. (2007). A scoring system to evaluate physical condition and quality of life in geriatric zoo mammals. Animal Welfare, 16, 309-318.

Fraser, D. (2009). Assessing animal welfare: Different philosophies, different scientific approaches. Zoo Biology, 28, 507-518.

Hare, V., Ripsky, D., Batterhill, R., Bacon, K., Hawk, K., \& Swaisgood, R. (2003). Giant panda enrichment: Meeting everyone's needs. Zoo Biology, 22, 401-416.

Harley, H. E., Fellner, W., \& Stamper, M. A. (2010). Cognitive research with dolphins (Tursiops truncatus) at Disney's The Seas: A program for enrichment, science, education, and conservation. International Journal of Comparative Psychology, 23, 331-343.

Hosey, G. R. (2000). Zoo animals and their human audiences: What is the visitor effect? Animal Welfare, 9, $343-357$.

Hosey, G. R. (2005). How does the zoo environment affect the behaviour of captive primates? Applied Animal Behaviour Science, 90, 107-129.

Hoy, J. M., Murray, P. J., \& Tribe, A. (2010a). Thirty years later: Enrichment practices for captive mammals. Zoo Biology, 29, 303-316.

Hoy, J. M., Murray, P. J., \& Tribe, A. (2010b). The potential for microchip-automated technology to improve enrichment practices. Zoo Biology, 29, 586-599.

Huber, H. F., \& Lewis, K. P. (2011). An assessment of gum-based environmental enrichment for captive gummivorous primates. Zoo Biology, 30, 71-78.

Hutchins, M., \& Smith, B. (2003). Characteristics of a world-class zoo or aquarium in the $21^{\text {st }}$ century. International Zoo Yearbook, 38, 130-141.

Izzo, G. N., Bashaw, M. J., \& Campbell, J. B. (2011). Enrichment and individual differences affect welfare indicators in squirrel monkeys (Saimiri sciureus). Journal of Comparative Psychology, 125, 347-352.

Jacobs, R. M., Ross, S. R., Wagner, K. E., Leahy, M., Meiers, S. T., \& Santymire, R. M. (2014). Evaluating the physiological and behavioral response of a male and female gorilla (Gorilla gorilla gorilla) during an introduction. Zoo Biology, 33, 394-402.

Johnston, J. M. (1979). On the relation between generalization and generality. The Behavior Analyst, 2, 1-6.

Kanaan, V. T., \& Hötzel, M. J. (2011). Letter to the editor: Does environmental enrichment really matter?: A case study using the eastern fence lizard, Sceloporus undulatus. Applied Animal Behaviour Science, 135, 169170.

Keen, H. A., Nelson, O. L., Robbins, C. T., Evans, M., Shepherdson, D. J., \& Newberry, R. C. (2014). Validation of a novel cognitive bias task based on difference in quantity of reinforcement for assessing environmental enrichment. Animal Cognition, 17, 529-541.

Koene, P. (2013). Behavioral ecology of captive species: Using behavioral adaptations to assess and enhance welfare of nonhuman zoo animals. Journal of Applied Animal Welfare Science, 16, 360-380.

Kreger M. D., Hutchins, M., \& Fascione, N. (1998). Context, ethics, and environmental enrichment in zoos and aquariums. In D. J. Shepherdson, J. Mellen, \& M. Hutchins (Eds.), Second nature: Environmental enrichment for captive animals. Washington, DC: Smithsonian Institution Press.

Kuczaj, S., Lacinak, T., Fad, O., Trone, M., Solangi, M., \& Ramos, J. (2002). Keeping environmental enrichment enriching. International Journal of Comparative Psychology, 15, 127-137.

Kuhar, C. W. (2008). Group differences in captive gorillas' reaction to large crowds. Applied Animal Behaviour Science, 110, 377-385. 
Kumar, V., Reddy, V. P., Kokkiligadda, A., Shivaji, S., \& Umapathy, G. (2014). Non-invasive assessment of reproductive status and stress in captive Asian elephants in three south Indian zoos. General and Comparative Endocrinology, 201, 37-44.

Kutska, D. (2009). Variation in visitor perceptions of a polar bear enclosure based on the presence of natural vs. unnatural enrichment items. Zoo Biology, 28, 292-306.

Law, G., \& Reid, A. (2010). Enriching the lives of bears in zoos. International Zoo Yearbook, 44, 65-74.

Lawson, D. P., Ogden, J., \& Snyder, R. J. (2008). Maximizing the contribution of science in zoos and aquariums: Organizational models and perceptions. Zoo Biology, 27, 458-469.

Maloney, M. A., Leighty, K. A., Kuhar, C. W., \& Bettinger, T. L. (2011). Behavioral responses of silverback gorillas (Gorilla gorilla gorilla) to videos. Journal of Applied Animal Welfare Science, 14, 96-108.

Maple, T. L. (2007). Toward a science of welfare for animals in the zoo. Journal of Applied Animal Welfare Science, $10,63-70$

Maple, T. L. (2013). The empirical zoo director - Building a foundation of evidence-based welfare and wellness: Keynote. Journal of Applied Animal Welfare Science, 16, 382.

Margulis, S. W., \& Westhus, E. J. (2008). Evaluation of different observational sampling regimes for use in zoological parks. Applied Animal Behaviour Science, 110, 363-376.

Mason, G., Clubb, R., Latham, N., \& Vickery, S. (2007). Why and how should we use environmental enrichment to tackle stereotypic behavior? Applied Animal Behaviour Science, 102, 163-188.

Mason, G. J., \& Latham, N. R. (2004). Can’t stop, won't stop: Is stereotypy a reliable animal welfare indicator? Animal Welfare, 13, S57-S69.

McPhee, M. E., Foster, J. S., Sevenich, M., \& Saunders, C. D. (1998). Public perceptions of behavioral enrichment: Assumptions gone awry. Zoo Biology 17, 525-534.

Meehan, C. L., \& Mench, J. A. (2007). The challenge of challenge: Can problem solving opportunities enhance animal welfare? Applied Animal Behaviour Science, 102, 246-261.

Mehrkam, L. R., \& Dorey, N. R. (2014). Is preference a predictor of enrichment efficacy in Galapagos Tortoises (Chelonoidis nigra)? Zoo Biology, 33, 275-284.

Melfi, V. (2013). Is training zoo animals enriching? Applied Animal Behaviour Science, 147, $299-305$.

Melfi, V. (2014). In response to the Letter to the Editor regarding the article: "Is training zoo animals enriching?" Applied Animal Behaviour Science, 152, 105-105.

Melfi, V., \& Hosey, G. (2011). Capacity building for better animal welfare. International Zoo Yearbook, 45, $274-281$.

Mellen, J., \& MacPhee, M. S. (2001). Philosophy of environmental enrichment: Past, present, and future. Zoo Biology, 20, 211-226.

Mendl, M., \& Paul, E. D. (2004). Consciousness, emotion, and animal welfare: Insights from cognitive science. Animal Welfare, 13, S17-S25.

Mendl, M., Burman, O. H. P., Parker, R. M. A., \& Paul, E. S. (2009). Cognitive bias as an indicator of animal emotion and welfare: Emerging evidence and underlying mechanisms. Applied Animal Behaviour Science, 118, 161181.

Miller, L. J. (2012). Visitor reaction to pacing behavior: Influence on the perception of animal care and interest in supporting zoological institutions. Zoo Biology, 31, 242-248.

Morimura, N. (2003). A note on enrichment for spontaneous tool use by chimpanzees. Applied Animal Behaviour Science, 82, 241-247.

Narayan, E. J., Webster, K., Nicolson, V., Mucci, A., \& Hero, J. (2013). Non-invasive evaluation of physiological stress in an iconic Australian marsupial: The Koala (Phascolarctos cinereus). General and Comparative Endocrinology, 187, 39-47.

Newberry, R. C. (1995). Environmental enrichment: Increasing the biological relevance of captive environments. Applied Animal Behaviour Science, 44, 229-243.

Pepperberg, I. M. (2004). Cognitive and communicative capacities of Grey parrots - implications for the enrichment of many species. Animal Welfare, 13, S203-S208.

Pomerantz, O., \& Terkel, J. (2009). Effects of positive reinforcement training techniques on the psychological welfare of zoo-housed Chimpanzees (Pan troglodytes). American Journal of Primatology, 71, 687-695.

Posta, B., Huber, R., \& Moore, D. E. (2013). The effects of housing on zoo elephant behavior: A quantitative case study of diurnal and seasonal variation. International Journal of Comparative Psychology, 26, 37-52.

Posta, B., Leighty, K. A., Alligood, C. A., \& Carlstead, K. (2013). Using science to understand elephant welfare. Journal of Applied Animal Welfare Science, 16, 395-396.

Quirke, T., \& O'Riordan, R. M. (2013). Evaluation and interpretation of the effects of environmental enrichment utilizing varying degrees of sampling effort. Zoo Biology, 32, 262-268. 
Rafacz M. L., \& Santymire, R. M. (2014). Using odor cues to elicit a behavioral and hormonal response in zoo-housed African wild dogs. Zoo Biology, 33, 144-149.

Reade, L. S., \& Waran, N. K. (1996). The modern zoo: How do people perceive zoo animals? Applied Animal Behaviour Science, 47, 109-118.

Reading, R. P., Miller, B., \& Shepherdson, D. (2013). The value of enrichment to reintroduction success. Zoo Biology, 32, 332-341.

Robins, J. G., \& Waitt, C. D. (2011). Improving the welfare of captive Macaques (Macaca sp.) through the use of water as enrichment. Journal of Applied Animal Welfare Science, 14, 75-84.

Rosier, R. L., \& Langkilde, T. (2011a). Does environmental enrichment really matter?: A case study using the eastern fence lizard, Sceloporus undulatus. Applied Animal Behaviour Science, 131, 71-76.

Rosier, R. L., \& Langkilde, T. (2011b). In response to the letter to the editor regarding the article: Does environmental enrichment really matter?: A case study using the eastern fence lizard, Sceloporus undulatus. Applied Animal Behaviour Science, 135, 171-172.

Ross, S. R., Lonsdorf, E. V., \& Stoinski, T. (2007). Letter the Editor: Assessing the welfare implications of visitors in a zoo setting: A comment on Wells (2005). Applied Animal Behaviour Science, 102, 130-133.

Ross, S. R., Schapiro, S. J., Hau, J., \& Lukas, K. E. (2009). Space use as an indicator of enclosure appropriateness: A novel measure of captive animal welfare. Applied Animal Behaviour Science, 121, 42-50.

Schapiro, S. J., \& Lambeth, S. P. (2007). Control, choice, and assessments of the value of behavioral management to nonhuman primates in captivity. Journal of Applied Animal Welfare Science, 10, 39-47.

Schetini de Azevedo, C., Cipreste, C. F., \& Young, R. J. (2007). Environmental enrichment: A GAP analysis. Applied Animal Behaviour Science, 102, 329-343.

Scott, E. M., Fitzpatrick, J. L., Nolan, A. M., Reid, J., \& Wiseman, M. L. (2003). Evaluation of welfare state based on interpretation of multiple indices. Animal Welfare, 12, 457-468.

Scott, E. M., Nolan, A. M., Reid, J., \& Wiseman-Orr, M. L. (2007). Can we really measure animal quality of life?: Methodologies for measuring quality of life in people and other animals. Animal Welfare, 16(S), 17-24.

Shabani, D. B., Wilder, D. A., \& Flood, W. A. (2001). Reducing stereotypic behavior through discrimination training, differential reinforcement of other behavior, and self-monitoring. Behavioral Interventions, 16, 279-286.

Shepherdson, D. J. (2003). Environmental enrichment: past, present, and future. International Zoo Yearbook, 38, 118124.

Shepherdson, D. J., Carlstead, K. C., \& Wielebnowski, N. (2004). Cross-institutional assessment of stress responses in zoo animals using longitudinal monitoring of faecal corticoids and behaviour. Animal Welfare, 13, S105S113.

Shepherdson, D. J., Lewis, K. D., Carlstead, K., Bauman, J., \& Perrin, N. (2013). Individual and environmental factors associated with stereotypic behavior and fecal glucocorticoid metabolite levels in zoo housed polar bears. Applied Animal Behaviour Science, 147, 268-277.

Shyne, A. (2006). Meta-analytic review of the effects of enrichment on stereotypic behavior in zoo mammals. Zoo Biology, 25, 317-337.

Siegford, J. M. (2013). Multidisciplinary approaches and assessment techniques to better understand and enhance zoo nonhuman animal welfare. Journal of Applied Animal Welfare Science, 16, 300-318.

Sidman, M. (1960). The reliability and generality of data. In Tactics of Scientific Research (pp. 42-67). Boston, MA: Authors Cooperative, Inc.

Smith, K. N., \& Kuhar, C. W. (2010). Siamangs (Hylobates syndactylus) and White-cheeked gibbons (Hylobates leucogenys) show few behavioral differences related to zoo attendance. Journal of Applied Animal Welfare Science, 13, 154-163.

Stoinski, T. S., Jaicks, H. F., \& Drayton, L. A. (2012). Visitor effects on the behavior of captive western lowland gorillas: The importance of individual differences in examining welfare. Zoo Biology, 31, 586-599.

Swaisgood, R. R. (2007). Current status and future directions of applied behavioural research for animal welfare and conservation. Applied Animal Behaviour Science, 102, 139-162.

Swaisgood, R. R., \& Shepherdson, D. J. (2005). Scientific approaches to enrichment and stereotypies in zoo animals: What's been done and where should we go next? Zoo Biology, 24, 499-518.

Swaisgood, R. R., Zhou, X., Zhang, G., Lindburg, D. G., \& Zhang, H. (2003). Application of behavioral knowledge to conservation in the giant panda. International Journal of Comparative Psychology, 16, 65-84.

Szokalski, M. S., Litchfield, C. A., \& Foster, W. K. (2012). Enrichment for captive tigers (Panthera tigris): Current knowledge and future directions. Applied Animal Behaviour Science, 139, 1-9.

Tarou, L. R., \& Bashaw, M. J. (2007). Maximizing the effectiveness of environmental enrichment: Suggestions from the experimental analysis of behavior. Applied Animal Behaviour Science, 102, 189-204. 
Tetley, C. L., \& O’Hara, S. J. (2012). Ratings of animal personality as a tool for improving the breeding, management and welfare of zoo mammals. Animal Welfare, 21, 463-476.

Wells, D. L. (2005). A note on the influence of visitors on the behaviour and welfare of zoo-housed gorillas. Applied Animal Behaviour Science, 93, 13-17.

Westlund, K. (2014a). Is training zoo animals enrichment?: A letter to the editor. Applied Animal Behaviour Science, $152,100-102$.

Westlund, K. (2014b). Training is enrichment - and beyond. Applied Animal Behaviour Science, 152, 1-6. 